Sitientibus Série Ciências Físicas 10: 1 -8 (2014)

\title{
Dinâmica de Campos Térmicos Aplicada ao Estudo da Superposição de Estados Coerentes e Estados de Fock via Função de Wigner
}

\author{
Thermofield Dynamics Applied to the Study of the Superposition of \\ Coherent States and Fock States through Wigner Function. \\ Lourival Manoel da Silva Filhd* \\ Departamento de Ciências Exatas e da Terra - DCET II - UNEB \\ Alagoinhas - BA - 40030-010
}

\begin{abstract}
A estrutura algébrica da dinâmica de campos térmicos, o formalismo da teoria quântica de campos a temperatura finita e a tempo real, são usados para o estudo do estado composto pela superposição de um estado número de Fock, $\mid n>$, com um estado coerente, $\mid \alpha>$. A análise dos efeitos da temperatura é realizada considerando a matriz densidade e a função de Wigner. Para tal propósito, vamos considerar a duplicação do estado original com o estado de vácuo do espaço dual.
\end{abstract}

Palavras-chaves: TQC; Temperatura Finita; Estado Coerente; Estado de Fock; Função de Wigner.

The algebraic structure of the dynamics of thermal fields, the formalism of quantum field theory at finite temperature and at real-time, are used in the study of the composite state as a superposition of a (number) Fock state, $|n\rangle$, and a coherent state, $|\alpha\rangle$. The analysis of the temperature effects is done taking into account the density matrix and the Wigner function. For such a purpose we will consider the duplication of the original state with the vacuum state of the dual space.

\section{Key-words: QFT; Finite Temperature; Coherent State; Fock State; Wigner} Function.

\section{INTRODUÇÃO}

Os efeitos térmicos em teorias de campos desempenham um papel relevante em diversas situações de interesse experimental e teórico, como em oscilações de íons pesados em física de altas energias e em quebra espontânea de simetria [1-4], ou nas flutuações do estado de vácuo do campo eletromagnético que levam ao efeito de Casimir [5] 8 , ou ainda em estados coerentes da radiação eletromagnética [911. De forma geral, os métodos teóricos introduzidos no contexto da física térmica procuram explorar as técnicas desenvolvidas para teorias à temperatura nula. Neste sentido, o método de tempo imaginário de Matsubara, para situações de equilíbrio, é um dos mais

*Endereço Eletrônico: lourivalmsfilho@uol.com.br celebrados [2]. Uma versão desse formalismo, estruturada na noção de espaço vetorial e a tempo real, foi proposta por Takahashi e Umezawa, conhecida como Dinâmica de Campos Térmicos, DCT, 12 14, e tem sido aplicada para tratar o efeito térmico em diversos fenômenos, em particular, sistemas bosônicos, posto que estes desempenham um papel importante na produção e detecção de condensados de Bose-Einstein, e as técnicas experimentais de laser cooling, armadilhas magnéticas e magnético-opticas se desenvolveram em grande medida [1, 2]. Estes aspectos, por outro lado, têm conduzido ao desenvolvimento experimental de produção de gás de Fermi degenerado, bem como condensados de isótopos raros [25, 7]. Esta situação sugere o estudo cuidadoso da noção de estados de Fermi coerentes e dos operadores densidades fermiônicos; alguns desses conceitos foram introduzidos para férmions há algum tempo [8 11]. Entretanto, 
somente mais recentemente Cahill e Glauber [12] discutiram noções como a função $P$, a função $Q$ e a função de Wigner no contexto de férmions. Esses elementos são introduzidos em contrapartida aos sistemas bosônicos, fato que se torna possível com o uso de variáveis grassmannianas.

Também constatamos na literatura grande interesse no estudo das propriedades de emaranhamento de sistemas multipartites [13, 14. A existência de emaranhamento está diretamente relacionada à natureza quântica do formalismo, estruturado sobre o espaço de Hilbert e o princípio de superposição. O presente interesse em estados emaranhados é fortemente condicionado pelo entendimento de que este é um ingrediente central em teletransporte, que por sua vez é um elemento estrutural da computação quântica [15, 16].

Neste trabalho, vou analisar o comportamento de um sistema composto pela superposição de estados coerentes com estados números de Fock, empregando a função de Wigner [6], quando submetido a um banho térmico. Tal análise ocorrerá a partir da duplicação apenas com o vácuo do espaço til, dual.

\section{DINÂMICA DE CAMPOS TÉRMICOS}

Nesta seção, apresentarei uma breve exposição dos princípios da Dinâmica de Campos Térmicos, (DCT), desenvolvida por Takahashi e Umezawa, sem maiores detalhes técnicos em seu aspecto matemático.

Para um sistema no equilíbrio térmico e considerando o ensemble canônico, a média para um observável arbitrário $A$ é definida como:

$$
\langle A\rangle=\frac{1}{Z(\beta)} \operatorname{Tr}[\exp (-\beta H) A]
$$

com

$$
\beta=\frac{1}{k_{b} T},
$$

onde $\operatorname{Tr}, k_{b}, T$, e $H$ são, respectivamente, o traço, a constante de Boltzmann, a temperatura, a função de partição e o hamiltoniano do sistema. A média de $A$ pode ser obtida usando a base dos autoestados do hamiltoniano, $|n\rangle$,

$$
\begin{aligned}
H|n\rangle & =E_{n} \mid n>, \\
<m \mid n> & =\delta_{m, n},
\end{aligned}
$$

e

$$
\sum_{n=0}^{\infty}|n><n|=1
$$

sendo $E_{n}$, a energia associada ao estado $|n\rangle$, e $\delta_{m, n}$, a função delta de Kronecker. Podemos reescrever (1) usando esta base, dita de Fock,

$$
\begin{aligned}
\langle A\rangle & =Z^{-1}(\beta) \sum_{(n)}\langle n|\exp (-\beta H) A| n\rangle \\
& =Z^{-1}(\beta) \sum_{(n)} \exp \left(-\beta E_{n}\right)\langle n|A| n\rangle .
\end{aligned}
$$

Como queremos o valor médio do operador em um estado termalizado, definido em um espaço de Hilbert apropriado, temos

$$
\begin{aligned}
\langle A\rangle & =\langle 0(\beta)|A| 0(\beta)\rangle \\
& =Z^{-1}(\beta) \sum_{(n)} \exp \left(-\beta E_{n}\right)\langle n|A| n\rangle .
\end{aligned}
$$

Aplicando a relação de completeza ao estado $\mid 0(\beta)>$,

$$
\left|0(\beta)>=\sum_{(n)}\right| n><n\left|0(\beta)>=\sum_{(n)} g_{n}(\beta)\right| n>,
$$

onde $g_{n}(\beta)=<n \mid 0(\beta)>$ é uma função escalar. Logo, podemos escrever a média de $A$ como

$$
\begin{aligned}
& \langle 0(\beta)|A| 0(\beta)\rangle \\
= & \sum_{(n, m)}\langle 0(\beta)|n><n| A|m><m| 0(\beta)\rangle \\
= & g_{n}^{*}(\beta) g_{m}(\beta)<n|A| m>.
\end{aligned}
$$

Para que a Eq. (9) satisfaça a condição em (7), o produto das funções escalares deve ser

$$
g_{n}^{*}(\beta) g_{m}(\beta)=Z^{-1}(\beta) \sum_{(n, m)} \exp \left(-\beta E_{n}\right) \delta_{m, n} .
$$


Como na equação acima, em seu lado direito, temos a função delta de Kronecker, ela sugere um produto escalar de vetores. Para justificar a igualdade, considera-se que o ente leva informações de dois espaços vetoriais distintos, sendo um, o espaço de Hilbert original e um outro, a determinar. Como as propriedades da base de Fock têm de ser preservadas, propõe-se para o segundo espaço a base $\{|\tilde{n}\rangle\}$ tal que

$$
\begin{aligned}
\tilde{H}|\tilde{n}\rangle & =E_{n} \mid \tilde{n}>, \\
<\tilde{n} \mid m> & =\delta_{m, n},
\end{aligned}
$$

e

$$
\sum_{n=0}^{\infty}|\tilde{n}><\tilde{n}|=1,
$$

onde $\tilde{H}$ é o hamiltoniano de um sistema fictício, sistema til ou dual, que tem como autovetores os elementos da base $\{|\tilde{n}\rangle\}$ e, como autovalor, $E_{n}$, idêntico ao autovalor da Eq. (3). Desta forma o ente $\mid 0(\beta)>$ é um elemento de um espaço de Hilbert, cuja base é formada pelo produto tensorial dos elementos da base $\{\mid \tilde{n}>\}$ pelos elementos da base do espaço original, $\{\mid n>\}$,

$$
g_{n}(\beta)=f_{n}(\beta) \mid \tilde{n}>,
$$

e

$$
\left|0(\beta)>=\sum_{n=0}^{\infty}\right| n>\otimes f_{n}\left|\tilde{n}>=\sum_{n=0}^{\infty} f_{n}\right| n, \tilde{n}>.
$$

Então,

$$
\begin{aligned}
\langle A\rangle & =\langle 0(\beta)|A| 0(\beta)\rangle \\
& =\sum_{m, n} f_{n}^{*}(\beta) f_{m}(\beta)\langle n, \tilde{n}|A| m, \tilde{m}> \\
& =\sum_{m, n} f_{n}^{*}(\beta) f_{m}(\beta) \delta_{m, n}\langle n,|A| m> \\
& =\sum_{n} f_{n}^{*}(\beta) f_{n}(\beta)\langle n|A| n>,
\end{aligned}
$$

onde o operador $A$ atua apenas no espaço de
Hilbert original. Comparando com a Eq. (6),

$$
f_{n}^{*}(\beta) f_{n}(\beta)=\frac{1}{Z(\beta)} \exp \left(-\beta E_{n}\right),
$$

e o estado $\mid 0(\beta)>$, vácuo termalizado, é descrito por:

$$
\left|0(\beta)>=\frac{1}{\sqrt{Z(\beta)}} \sum_{(n)} \exp \left(-\beta \frac{E_{n}}{2}\right)\right| n, \tilde{n}>.
$$

Temos, então, o estado de vácuo termalizado no espaço de Hilbert duplicado que, como se percebe, é constituído por quanta, $|n, \tilde{n}\rangle$.

A dinâmica de campos térmicos, DCT, é então introduzida por dois ingredientes básicos: primeiro, considerando a álgebra dos operadores de von Neumann no espaço de Hilbert, existe um conjunto de operadores que comutam introduzidos por uma conjugação modular; isto corresponde a uma duplicação do espaço de Fock original do sistema, levando-o ao espaço expandido $H_{T}=H \otimes \tilde{H}$. A conjugação modular é expressa pelo mapeamento $\tilde{:} H \rightarrow \tilde{H}$, associando a cada operador $A$ atuando sobre o espaço $H$ com dois operadores em $H_{T}, A$ e $\tilde{A}$, que estão conectados por uma conjugação modular em uma $c^{*}$-álgebra:

$$
\begin{aligned}
\left(A_{i} A_{j}\right)^{\tilde{r}} & =\tilde{A}_{i} \tilde{A}_{j}, \\
\left(c A_{i}+A_{j}\right)^{\tilde{2}} & =c^{*} \tilde{A}_{i}+\tilde{A}_{j}, \\
\left(A_{i}^{\dagger}\right)^{\tilde{2}} & =\left(\tilde{A}_{i}\right)^{\dagger}, \\
\left(\tilde{A}_{i}\right)^{\tilde{y}} & =-\epsilon A_{i},
\end{aligned}
$$

com $\epsilon=-1$ para bósons e $\epsilon=+1$ para férmions. Em DCT, a conjugação modular é também chamada de normas de conjugação til. As variáveis til, definidas nos comutadores da álgebra de von Neumann estão associados com os geradores do grupo modular dados por $\hat{A}=A-\tilde{A}$. Com esses elementos, as representações redutíveis do grupo de Lie podem ser estudadas; em particular, simetrias cinemáticas tais como, o grupo de Lorentz. Isto dá lugar a equação de movimento tipo Liouvillevon Neumann. O segundo ingrediente básico é uma transformação de Bogoliubov, $B(\alpha)$, introduzindo uma rotação nas variáveis til e não- 
til, tais que os efeitos térmicos emirjam de um estado condensado. O parâmetro de rotação $\alpha$ está associado à temperatura, e este procedimento é equivalente à média térmica estatística usual. Em uma notação duplicada tradicional,

$$
A^{r}(\alpha)=\left(\begin{array}{c}
A(\alpha) \\
\epsilon \tilde{A}^{\dagger}(\alpha)
\end{array}\right)=B(\alpha)\left(\begin{array}{c}
A \\
\epsilon \tilde{A}^{\dagger}
\end{array}\right)
$$

$\operatorname{com}\left(A^{r}(\alpha)\right)^{\dagger}=\left(A^{\dagger}(\alpha), \epsilon \tilde{A}(\alpha)\right)$, e a transformada de Bogoliubov dada por

$$
B(\alpha)=\left(\begin{array}{cc}
u(\alpha) & -v(\alpha) \\
\epsilon v(\alpha) & u(\alpha)
\end{array}\right)
$$

onde $u^{2}(\alpha)+\epsilon v^{2}(\alpha)=1$.

A parametrização da transformação de Bogoliubov em DCT é obtida fazendo $\alpha=\beta=$ $T^{-1}$, e por estabelecer que a média do operador número termalizado, $N(\alpha)=a^{\dagger}(\alpha) a(\alpha)$, isto é $\langle N(\alpha)\rangle_{\alpha}=\left\langle 0, \tilde{0}\left|a^{\dagger}(\alpha) a(\alpha)\right| 0 \tilde{0}\right\rangle$, fornece a distribuição de Bose ou de Fermi, ou seja,

$$
N(\alpha)=v^{2}(\beta)=(\exp (\beta \epsilon)+\xi)^{-1} .
$$

Por simplicidade de notação, temos usado $A \equiv$ $a$ e $\tilde{A}=\tilde{a}$, e

$$
a(k)=u(\alpha) a(k, \alpha)+v\left(\alpha \tilde{a}^{\dagger}(k, \alpha),\right.
$$

tal que os outros operadores $\left(a^{\dagger}(k), \tilde{a}(k), \tilde{a}^{\dagger}(k)\right)$ são obtidos ao se aplicar o hermitiano ou as regras de conjugação til. É então mostrado que a média térmica, $\langle N(\alpha)\rangle_{\alpha}$ pode ser escrita como $\langle N(\alpha)\rangle_{\alpha}=\left\langle 0(\alpha)\left|a^{\dagger} a\right| 0(\alpha)\right\rangle$, onde $\mid 0(\alpha)>$ é dado por $|0(\alpha)\rangle=U(\alpha)|0, \tilde{0}\rangle$, com

$$
U(\alpha)=\exp \left(\theta(\alpha)\left[a^{\dagger} \tilde{a}^{\dagger}-a \tilde{a}\right]\right)
$$

O espaço de Hilbert é constituído dos estados $\beta,|0(\beta)>=U(\beta)| 0, \tilde{0}>\mathrm{com}$

$$
|0, \tilde{0}>=\underbrace{\otimes}_{\kappa}| 0, \tilde{0}>_{\kappa},
$$

e $\mid 0, \tilde{0}>_{\kappa}$ é o vácuo para o modo $\kappa$. Então,

$$
\begin{aligned}
a(\kappa, \beta) \mid 0(\beta)> & =\tilde{a}(\kappa, \beta) \mid 0(\beta)>, \\
\mathrm{e} & \\
<0(\beta) \mid 0(\beta)> & =1 .
\end{aligned}
$$

Isto mostra que $\mid 0(\beta)>$ é um vácuo para os operadores termalizados $a(\kappa, \beta)$. Entretanto, ele é um condensado para os operadores $a$ e $a^{\dagger}$.

Considerando apenas o modo do campo, pode-se escrever $\mid 0(\beta)>$ em termos das funções $u(\beta)$ e $v(\beta)$ como segue

$$
\begin{aligned}
\mid 0(\beta)> & \left.=\frac{1}{u(\beta)} \exp \left[\frac{u(\beta)}{v(\beta)} a^{\dagger} \tilde{(} a\right)^{\dagger}\right] \mid 0, \tilde{0}>, \\
& =\frac{1}{u(\beta)} \sum_{(n)}\left[\frac{u(\beta)}{v(\beta)}\right]^{n} \mid n, \tilde{n}>.
\end{aligned}
$$

Definindo,

$$
\rho_{n n}^{1 / 2}=\frac{1}{u(\beta)} \sum_{(n)}\left[\frac{u(\beta)}{v(\beta)}\right]^{n},
$$

temos

$$
\left|0(\beta)>=\sum_{(n)} \rho_{n n}^{1 / 2}(\beta)\right| n, \tilde{n}>=\rho^{1 / 2}(\beta) \mid I>,
$$

$\operatorname{com}\left|I>=\sum_{(n)}\right| n, \tilde{n}>\mathrm{e}$

$$
\begin{aligned}
\rho(\beta) & =\frac{1}{|u(\beta)|^{2}}\left[\frac{|v(\beta)|^{2}}{|u(\beta)|^{2}}\right]^{a^{\dagger} a} \\
& =\frac{1}{|u(\beta)|^{2}} \sum_{n=0}^{\infty}\left[\frac{|v(\beta)|^{2}}{|u(\beta)|^{2}}\right]^{n}|n><n|,
\end{aligned}
$$

$\mathrm{Ou}$

$$
\begin{aligned}
\rho(\beta) & =\frac{1}{1+\tilde{n}(\beta)} \sum_{r=0}^{\infty}\left[\frac{\tilde{n}(\beta)}{1+\tilde{n}(\beta)}\right]^{r}|r><r|, \\
\tilde{n}(\beta) & =\frac{1}{\exp \beta \omega-1} .
\end{aligned}
$$

Assim, a média de um observável $A$ é dada por

$$
<0(\beta)|A| 0(\beta)>=\sum_{(n)} \rho_{n n}(\beta) A_{n n}=\operatorname{Tr}[\rho(\beta) A],
$$

A seguir temos exemplos de estados termalizados, o coerente e o número de Fock,

$$
\begin{aligned}
\mid \alpha, \beta> & =U(\beta) D(\alpha) \mid 0, \tilde{0}>=D(\alpha, \beta) \\
& =\exp \left[\alpha a^{\dagger}(\beta)-\alpha^{*} a(\beta)\right] \mid 0(\beta)>,
\end{aligned}
$$


e $\left|n, \beta>=U(\beta) \frac{\left(a^{\dagger}\right)^{n}}{\sqrt{n !}}\right| 0, \tilde{0}>=\frac{\left(a^{\dagger}(\beta)\right)^{n}}{\sqrt{n !}} \mid 0(\beta)>$, com $D(\alpha)$ e $D(\alpha, \beta)$ sendo operadores unitários de deslocamentos não-termalizado e termalizado, respectivamente, e $\alpha$ um número complexo arbitrário; o termo "operador deslocamento" vem da propriedade

$$
D^{\dagger}(\alpha, \beta) a(\beta) D(\alpha, \beta)=a(\beta)+\alpha .
$$

Usando o fato que

$$
a(\beta)\left|0(\beta)>=\left[u(\beta) a+v(\beta) \tilde{a}^{\dagger}\right]\right| 0(\beta)>=0,
$$

os operadores termalizados podem ser descritos somente em termos de operadores não-til

$$
a^{\dagger}(\beta)\left|0(\beta)>=\frac{a^{\dagger}}{u(\beta)}\right| 0(\beta)>\text {. }
$$

Dessa forma, os estados coerente e número termalizados são

$$
\left|\alpha, \beta>=\exp \left(\frac{|\alpha|^{2}}{2}\right) \exp \left[\frac{\alpha a^{\dagger}}{u(\beta)}\right]\right| 0(\beta)>,
$$

$\mathrm{e}$

$$
\left|n, \beta>=\frac{1}{\sqrt{n !}}\left[\frac{a^{\dagger}}{u(\beta)}\right]^{n}\right| 0(\beta)>
$$

Para identificar a natureza física de tais estados térmicos, calculamos as correspondentes matrizes densidades

$$
\begin{aligned}
\rho_{\mid \alpha(\beta)>} & =\exp \left(-|\alpha|^{2}\right) \exp \left[\frac{\alpha a^{\dagger}}{\sqrt{1+\overline{\tilde{n}(\beta)}}}\right] \rho_{\beta} \times \\
& \times \exp \left[\frac{\alpha^{*} a}{\sqrt{1+\tilde{n}(\beta)}}\right],
\end{aligned}
$$

e

$$
\rho_{\mid n(\beta)>}=\frac{a^{\dagger} \rho_{\beta} a^{n}}{n ![1+\tilde{n}(\beta)]^{n}}
$$

Outros tipos de estados podem ser obtidos usando operadores til.

A seguir, investigarei propriedades não clássicas de estados térmicos introduzidos pela superposição de estados coerente com os de Fock, a partir da função de Wigner.

\section{PROPRIEDADES ESTATÍSTICAS} DE ESTADOS DE FÓTONS TÉRMICOS

\section{A. O Sistema e a Matriz Densidade}

Usando a estrutura da álgebra linear da DCT, exploro a superposição de estados com excesso de fótons. Seja a superposição

$$
\mid \psi(n, \alpha)>=\frac{1}{\sqrt{N}}(A|\alpha>+B| n>),
$$

onde o fator de normalização é

$$
N=A^{2}+2 \frac{A B \Re\left(\alpha^{n}\right) \exp \left(-\frac{1}{2}|\alpha|^{2}\right)}{\sqrt{n !}}+B^{2} .
$$

Realizando o produto tensorial com $\mid \tilde{0}>$,

$$
\mid \psi(\alpha, n, \tilde{0})>=\frac{1}{\sqrt{N}}(A|\alpha, \tilde{0}>+B| n, \tilde{0}>),
$$

que pode ser reescrita como segue

$$
\begin{aligned}
\mid \psi(\alpha, n, \tilde{0})> & =\frac{1}{\eta}\left[A \exp \left(-\frac{|\alpha|^{2}}{2}\right) \times\right. \\
& \left.\times \sum_{m=0}^{\infty} \frac{\alpha^{m}}{\sqrt{m !}}|m, \tilde{0}>+B| n, \tilde{0}>\right)
\end{aligned}
$$

com $\eta=1 / \sqrt{N}$. Usando a transformação de Bogoliubov e escrevendo os vetores em termos de vácuo do espaço duplicado, resulta 


$$
\mid \psi(\alpha, n, \tilde{0} ; \beta)>=\frac{1}{\eta}\left[A \exp \left(-\frac{|\alpha|^{2}}{2}\right) \sum_{m=0}^{\infty} \frac{\alpha^{m}}{\sqrt{m !}}\left(a^{\dagger}(\beta)\right)^{m}\left|0, \tilde{0} ; \beta>+B \frac{\left(a^{\dagger}(\beta)\right)^{\dagger}}{\sqrt{n !}}\right| 0, \tilde{0} ; \beta>\right] \text {. }
$$

Considerando $\rho(\beta)$ dado pelas equações $(25,26)$ matriz densidade pode ser descrita como e (31 32) após algumas operações algébricas, a

$$
\begin{aligned}
\rho_{\mid \psi(\alpha, n ; \beta)>} & =\frac{1}{N(1+\tilde{n}(\beta))} \sum_{r=0}^{\infty}\left[\frac{\tilde{n}(\beta)}{1+\tilde{n}(\beta)}\right]^{r}+\left\{\left[\sum_{m, m^{\prime}=0}^{\infty} \frac{A^{2} \alpha^{* m} \alpha^{m^{\prime}}}{m ! m^{\prime} ![u(\beta)]^{\left(m+m^{\prime}\right)}} \frac{\sqrt{\left(r+m^{\prime}\right) !(r+m) !}}{r !} \times\right.\right. \\
& \left.\times\left|r+m^{\prime}\right\rangle\left\langle r+m\left|+\frac{B^{2}(r+n) !}{n ! r !}\right| r+n\right\rangle\langle r+n|\right]+\sum_{m=0}^{\infty} \frac{A B \exp \frac{(-\alpha)^{2}}{2}}{[u(\beta)]^{(m+n)}} \frac{\sqrt{(r+m) !(r+n) !}}{m ! r ! \sqrt{n !}} \times \\
& \left.\times\left(\alpha^{m}|r+m\rangle\left\langle r+n\left|+\alpha^{* m}\right| r+n\right\rangle\langle r+m|\right)\right\} .
\end{aligned}
$$

\section{B. A Função de Wigner}

Uma das representações básicas de um estado modo campo no espaço de fase é a função de Wigner. Definida como a transformada de Weyl do operador densidade, o resultado dessa transformação é uma quase probabilidade e está associado ao caráter clássico ou quântico do sistema estudado: resultados positivos indicam que o sistema possui características clássicas, enquanto os negativos, características quânticas. A função de Wigner é

$$
\begin{aligned}
W(p, q) & =\frac{1}{(2 \pi \hbar)^{L}} \int_{-\infty}^{+\infty} d Q \exp \left(\frac{-i Q p}{\hbar}\right) \times \\
& \times\left\langle q+\frac{Q}{2}|\rho| q-\frac{Q}{2}\right\rangle
\end{aligned}
$$

onde $L$ representa o número de dimensões da integração, $\rho, p$ e $q$ são respectivamente, o operador densidade, o momentum e a coordenada espacial do sistema [17].

Para o sistema estudado, Eq.(36) e com a sua matriz densidade expressa pela eq. (37), após algumas operações algébricas, a função de Wigner assume a forma

$$
\begin{aligned}
& W(p, q ; \beta)=\frac{1}{N \pi \hbar(1+\tilde{n}(\beta))} \sum_{r=0}^{\infty} \frac{1}{r !}\left[\frac{\tilde{n}(\beta)}{1+\tilde{n}(\beta)}\right]^{r} \times \\
& \times\left\{w_{\alpha \alpha}+w_{n n}+w_{\alpha n}+w_{n \alpha}\right\}, \\
& \operatorname{com}
\end{aligned}
$$

$$
\begin{aligned}
w_{\alpha \alpha}= & A^{2} \sum_{m, m^{\prime}=0}^{\infty} \frac{\alpha^{* m}}{m !} \frac{\alpha^{m^{\prime}}}{m^{\prime} !} \frac{(-1)^{r+m^{\prime}}}{2^{\left(r+\frac{m+m^{\prime}}{2}\right)}} \frac{\exp \left[-\left(\frac{q^{2}}{b^{2}}-\tau\right)\right]}{[u(\beta)]^{m+m^{\prime}}} \times \\
\times & {\left[\begin{array}{c}
2^{(r+m)}\left(r+m^{\prime}\right) ! \gamma^{\left(m-m^{\prime}\right)} L_{r+m^{\prime}}^{m-m^{\prime}}\left[2|\gamma|^{2}\right], \text { para } m \geq m^{\prime} \\
2^{\left(r+m^{\prime}\right)}(r+m) !(-\gamma)^{\left(m^{\prime}-m\right)} L_{r+m}^{m^{\prime}-m}\left[2|\gamma|^{2}\right], \text { para } m<m^{\prime} ;
\end{array}\right.}
\end{aligned}
$$




$$
\begin{aligned}
w_{n n} & =\frac{B^{2}(-1)^{r+n} \exp (-|\gamma|)}{[u(\beta)]^{2 n} n !} L_{r+n}\left[2|\gamma|^{2}\right] ; \\
w_{\alpha n} & =A B \exp \left[-\left(\frac{|\alpha|^{2}}{2}\right)\right] \sum_{m=0}^{\infty} \frac{-(1)^{r+n} \alpha^{* m} \exp (-|\gamma|)^{2}}{m ! \sqrt{n !}[u(\beta)]^{m+n} 2^{r+\frac{m+n}{2}} \times} \\
& \times\left[\begin{array}{c}
2^{(r+m)}(r+n) ! \gamma^{(m-n)} L_{r+n}^{m-n}\left[2|\gamma|^{2}\right], \quad \text { para } n \leq m \\
2^{(r+n)}(r+m) !(-\gamma)^{(n-m)} L_{r+m}^{n-m}\left[2|\gamma|^{2}\right], \quad \text { para } n>m ;
\end{array}\right. \\
w_{n \alpha} & A B \exp \left[-\left(\frac{|\alpha|^{2}}{2}\right)\right] \sum_{m=0}^{\infty} \frac{(-1)^{r+m} \alpha^{m} \exp \left(-|\gamma|^{2}\right)}{m ! \sqrt{n !}[u(\beta)]^{m+n} 2^{r+\frac{m+n}{2}} \times} \\
\times & {\left[\begin{array}{c}
2^{(r+n)}(r+m) ! \gamma^{(n-m)} L_{r+m}^{n-m}\left[2|\gamma|^{2}\right], \quad \text { para } n \geq m \\
2^{(r+m)}(r+n) !(-\gamma)^{(m-n)} L_{r+n}^{m-n}\left[2|\gamma|^{2}\right], \quad \text { para } n<m ;
\end{array}\right.}
\end{aligned}
$$

onde

$\gamma=\tau+q / b, \quad-\gamma=\tau^{*}-q / b, \quad \tau=-i p b / \hbar$ $|\gamma|^{2}=(q / b)^{2}-\tau^{2}, \quad b=\sqrt{\hbar / m \omega}$.

Com esta função, faz-se o mapeamento do sistema no espaço de fase e, considerando que ela é uma quasi-probabilidade por con- ter também valores negativos, estes estão associados ao caráter quântico do sistema, enquanto seus valores positivos estão associados ao caráter clássico.

Alguns gráficos referentes à função de Wigner (39) estão ao final das referências.
[1] H.T.C. Stoof, M. Houbiers, C.A. Sackett, R.G. Hulet. Phys. Rev. Lett. 76, 10 (1996).

[2] C.J. Myatt, E.A. Burt, R W. Grist, E.A. Cornell, C.E. Wieman. Phys. Rev. Lett. 78, 586 (1997).

[3] E. Hodby, S.T. Thompson, C.A. Regal, M. Greiner, A.C. Wilson, D.S. Jin, E.A. Cornell, C.E. Wieman. arXiv:cond-mat/0411487, (2004).

[4] K.E. Strecker, G.B Partridge, R. G. Hulet. Phys. Rev. Lett. 91, 080406 (2003).

[5] J. Cubizolles, T. Bourdel, S.J.J.M.F. Kokkelmans, G.V. Shlyapnikov, C. Salomon. Phys. Rev. Lett. 91, 240401 (2003).

[6] H. Belich, L.M. Silva, J.A. Helayël-Neto, A.E. Santana. Phys. Rev. D 84, 045007-1 (2011).

[7] T. Bourdel, L. Khaykovich, J. Cubizolles, J. Zhang, F. Chevy, M. Teichmann, L. Tarruell, S.J.J.M.F. Kokkelmans, C. Salomon. Phys. Rev. Lett. 93, 050401 (2004).

[8] Y. Ohnuki, T. Kashiwa. Prog. Theor. Phys. 60, 548 (1978).

[9] F. Hong-yi. Phys. Rev. A 40, 4237 (1989).
[10] K. Svozil. Phys. Rev. Lett. 65, 3341 (1990).

[11] S. Chaturvedi R. Sandhya, V. Srinivasan, R. Simon. Phys. Rev. A 41, 3969 (1990).

[12] K.E. Cahill, R.J. Glauber. Phys. Rev. A 59, 1538 (1999).

[13] A. Shimony, Measures of Entanglement, in The Dilemma of Einstein, Podolsky and Rosen - 60 Years Later. Editors: A. Mann, M. Revzen. Bristol: IOP (1996).

[14] G. Alber, T. Beth, M. Horodecki, R. Horodeki, M. Rötteler, H. Weinfurter, R. Werner, A. Zeilinger, Quantum Information. Berlin: Springer-Verlag (2001).

[15] C.H. Bennett, Z. Brassard, C. Crépeau, R. Jozsa, A. Peres, W.K. Wootters. Phys. Rev. Lett. 70, 1895 (1993).

[16] D. Bouwmeester, J.W. Pan, K. Mattle, M. Eibl, H. Weinfurter, A. Zeilinger. Nature 390, 575 (1997).

[17] M.A. Marchiolli, Quantum Mechanics in Phase Space: I. The Weyl-Wigner Formalism. Rev. Bras. Ens. Fis. 24, (4) 421 (2002). 


\section{$\sigma=0,32, \alpha=1,0 \mathrm{n}=1$ e $\mathrm{T}=0 \mathrm{~K}$}

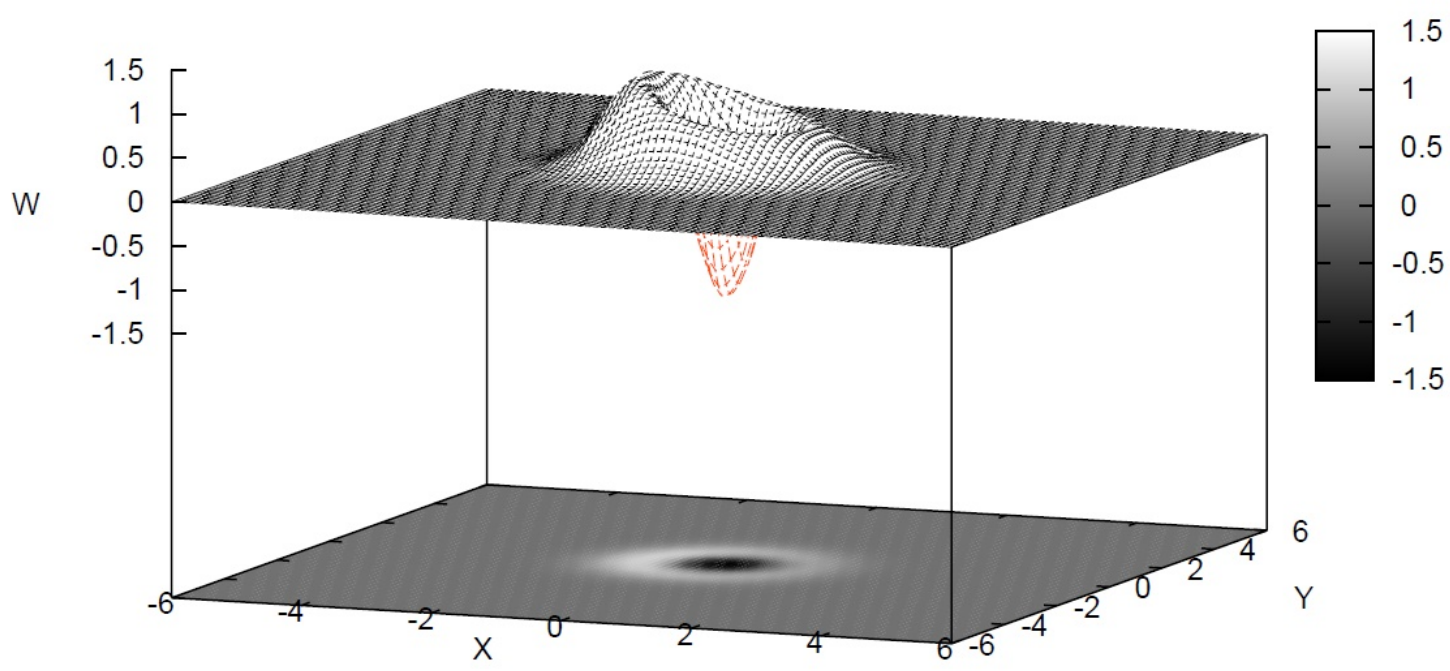

FIGURA 1: Função de Wigner: gráfico geral.

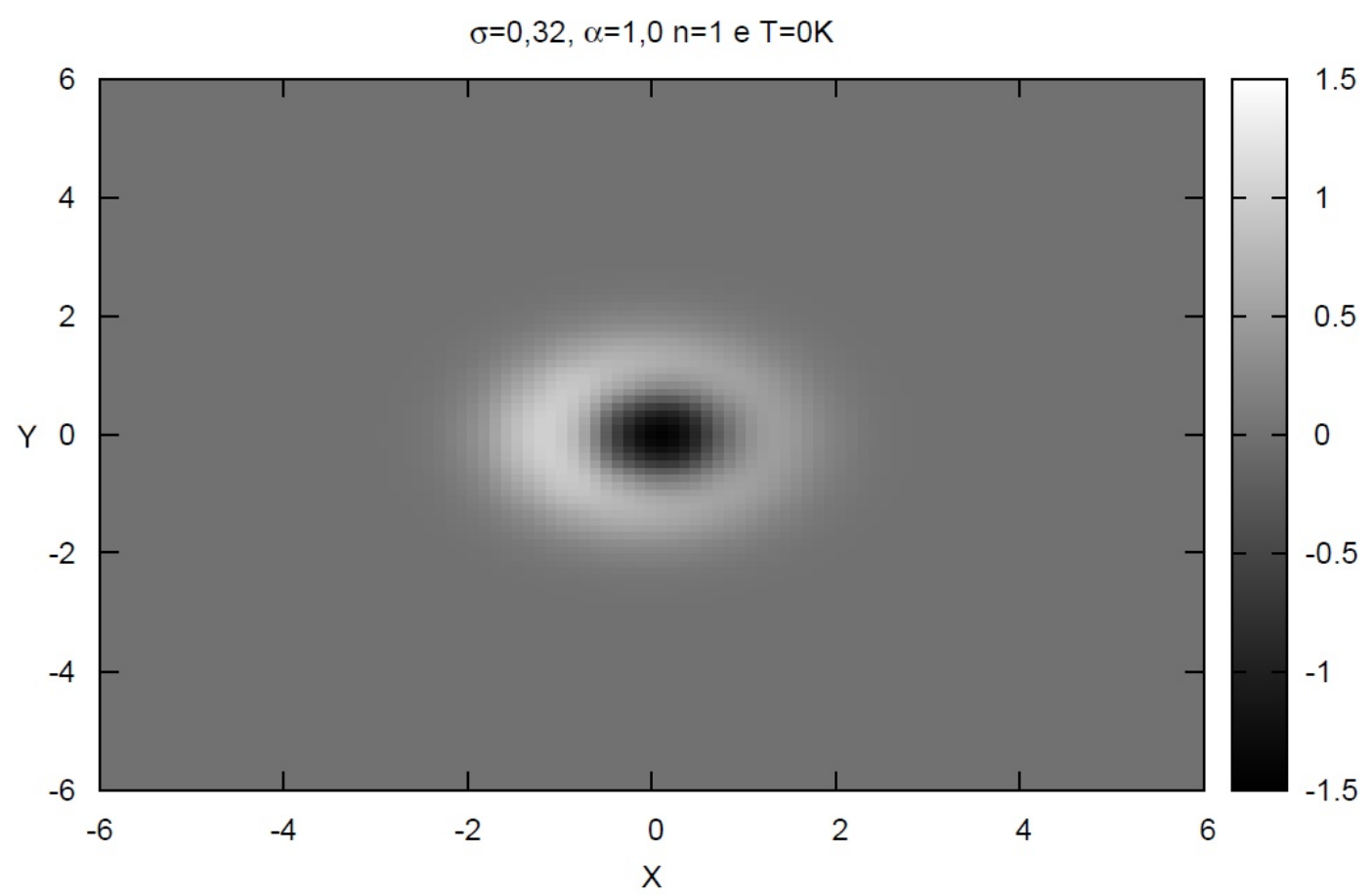

FIGURA 2: Função de Wigner: vista do plano XY. 